\title{
Software Antropmeter, una Nueva Herramienta para Análisis Facial
}

\author{
Antropmeter Software, a New Tool for Facial Analysis \\ "Iván Suazo Galdames;"Xaviera López Cortés \& **Valeria Márquez Miranda
}

\begin{abstract}
SUAZO, G. I.; LÓPEZ, C. X. \& MÁRQUEZ, M. V. Software Antropmeter, una nueva herramienta para análisis facial. Int. J. Morphol., 26(4):973-974, 2008.

RESUMEN: El análisis de las dimensiones y proporciones faciales es necesario en distintos ámbitos de la odontoestomatología y de la antropología física. En este informe presentamos el software Antropmeter, diseñado para realizar análisis de dimensiones y proporciones faciales, en base a fotografías estandarizadas, de fácil manejo por parte del clínico y de utilidad en análisis faciales estéticos y antropológicos.
\end{abstract}

PALABRAS CLAVE: Antropometría; Análisis facial; Proporciones faciales; Fotogrametría; Software.

\section{INTRODUCCIÓN}

La concepción de estética facial involucra principios artísticos básicos que relacionan los rasgos individuales en conjunto a la cara (Stough et al., 1997). Los análisis de las proporciones faciales tienen importancia en el análisis estético de la cara (Ricketts, 1982) y en la planificación o evaluación de intervenciones quirúrgicas cráneofaciales (Shell \& Woods, 2004, Edler et al., 2006).

Las proporciones del esqueleto craneofacial también han sido analizadas con fines antropológicos y forenses. Trujillo et al. (2007) y Suazo et al. (2008) describieron una serie de proporciones áuricas cráneofaciales que podrían ser utilizadas en la reconstrucción del cráneo con fines forenses.

La antropometría directa ha sido por largo tiempo el principal método para determinar las dimensiones de distintas estructuras anatómicas. En la actualidad, el método más utilizado es el de la antropometría indirecta, mediante fotografía y análisis computacional y, al respecto, Farkas et al. (1980) y Nechala et al., (1999) concluyeron que no existen diferencias significativas entre ambos métodos. Furnas (1987) describió que la estandarización de las fotografías con una regla graduada junto al paciente entrega una imagen en tamaño real.

En una evaluación piloto, Troncoso et al. (2008) realizaron mediciones y determinaron proporciones nasales utilizando un software denominado Antropmeter, desarrollado en la Universidad de Talca, Chile.
En el presente informe presentamos el software Antropmeter y lo colocamos a disposición de clínicos e investigadores para su uso y evaluación.

Software Antropmeter. Este software ha sido desarrollado por un esfuerzo conjunto de la Unidad de Anatomía de la Facultad de Ciencias de la Salud y de la Escuela de Bioinformática de la Facultad de Ingeniería, ambas de la Universidad de Talca, Chile.

Antropmeter es un software hecho para el cálculo de distancias y proporciones faciales del ser humano, en donde se encuentran los distintos puntos representativos de la cara.

Antropmeter realiza mediciones en base a una fotografía estandarizada de frente y de perfil (norma frontal y lateral), con una dimensión de 1088 x 816 pixeles. La distancia del foco al punto pronasal debe ser de $70 \mathrm{~cm}$. El paciente se ubica sentado, con la cabeza paralela al piso, en relación el plano de Frankfort (Troncoso et al.).

El software permite cargar una fotografía, marcar puntos anatómicos o cefelométricos de interés y determinar la distancia existente entre los puntos, por ejemplo endo-canthion derecho-endocanthion izquierdo (Fig. 1). El cálculo de medidas con Antropmeter es hecho en unidad de $\mathrm{cm}$.

\footnotetext{
* Unidad de Anatomía Normal, Facultad de Ciencias de la Salud, Universidad de Talca, Chile.

** Escuela de Bioinformática, Facultad de Ingeniería, Universidad de Talca, Chile.
} 


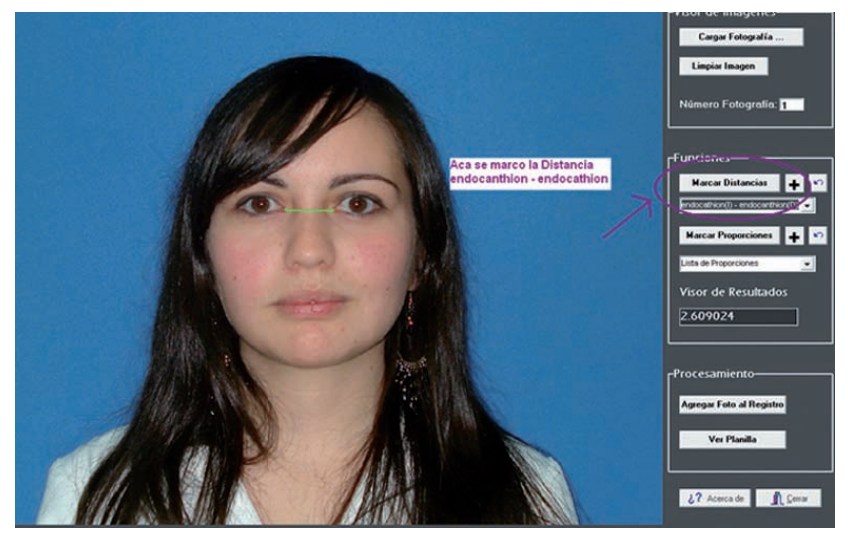

Fig 1. Ejemplo de la opción marcar distancias del software Antropmeter para determinar la distancia endocathion derechoencocanthion izquierdo

Además, a partir de los trazos formados por la unión de dos puntos permite analizar las relaciones de proporcionalidad entre ellos (Fig. 2).

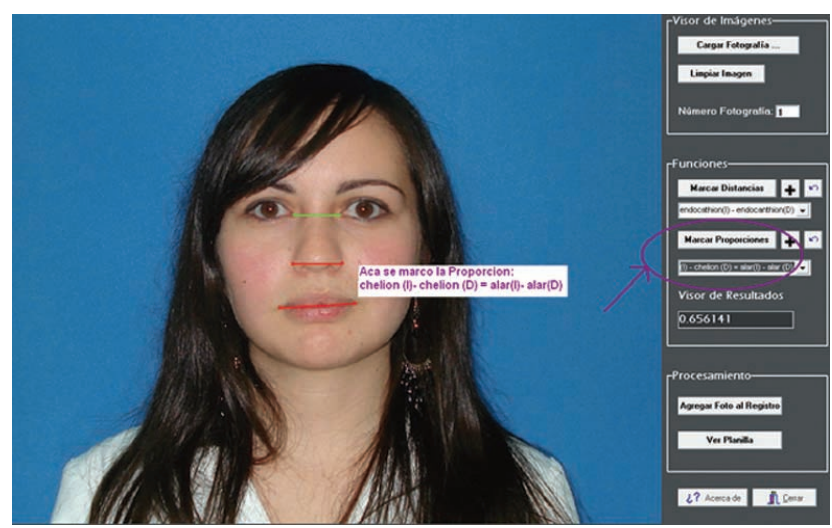

Fig 2. Ejemplo de la opción marcar proporciones del software Antropmeter para relacionar los trazos chelion derecho - chelion izquierdo con alar derecho - alar izquierdo.

\section{REFERENCIAS BIBLIOGRÁFICAS}

Edler, R.; Agarwal, P.; Wertheim, D. \& Greenhill, D. The use of anthropometric proportion indices in the measurement of facial attractiveness. Eur. J. Orthod., 28:274-81, 2006.

Farkas, L. G.; Bryson, W. \& Klotz, J. Is photogrammetry of the face reliable? Plast. Reconstr. Surg., 66(3):346-55, 1980.

Furnas, D. W. Precision nasal profileplasty with life-sized slide projections, calibrated xerograms, and intraoperative measurements. Clin. Plast. Surg., 14(4):631-7, 1987.

Nechala, P.; Mahoney, J. \& Farkas, L. G. Digital two-dimensional photogrammetry: a comparison of three techniques of obtaining digital photographs. Plast. Reconstr. Surg., 103(7):1819-25, 1999.
Una vez que el usuario ha recaudado todas las distancias y proporciones necesarias para su estudio, puede exportar sus resultados a una planilla Excel (Microsoft Office Excel 2003 o compatible), la que contiene 2 hojas "Planilla de Distancias" y "Planilla de Proporciones", cada cual realiza automáticamente el cálculo de los estadísticos descriptivos de las dimensiones y proporciones analizadas.

El software y un completo manual del usuario se encuentran disponibles (isuazo@utalca.cl)

SUAZO, G. I.; LÓPEZ, C. X. \& MARQUEZ, M. V. Antropmeter software, a new tools for facial analysis. Int. J. Morphol., 26(4):973$974,2008$.

SUMMARY: The dimensions and facial proportions analysis are necessary in different areas of the odontostomatology and physical anthropology practice. In this report we present the Antropmeter software, designed to carry out analysis of dimensions and facial proportions, based on standardized pictures, of easy handling on the part of the clinical one and of utility in aesthetic and anthropological facial analysis.

KEY WORDS: Anthropometry; Facial analysis; Facial proportions; Photogrametry; Software.

Ricketts, R. M. Divine proportion in facial esthetics. Clin. Plast. Surg., 9:401-22, 1982.

Shell, T. L. \& Woods, M. G. Facial aesthetics and the divine proportion: a comparison of surgical and non-surgical class II treatment. Aust. Orthod. J., 20:51-63, 2004.

Stough, D. B.; Schell, B. J. \& Weyrich, R. P. The role of facial proportion in hair restoration surgery. Ann. Plast. Surg., 38:12936, 1997.

Suazo, G. I. C.; Trujillo, H. E. G.; Cantín, L. M. G. \& Zavando, M. D. A. Determinación de proporciones áureas craneofaciales para la reconstrucción con fines de identificación médicolegal. Int. J. Morphol., 26(2):331-5, 2008.

Troncoso, J. Suazo, I. Cantín, M. \& Zavando, D. Sexual dimorphism in the nose morphotype in adult chilean. Int. J. Morphol., 26(3):537-42, 2008.

Trujillo, H. E. G.; Suazo, G. I. C.; Cantín, L. M. G \& Vargas, A. R. A. Determinación de nuevas proporciones áuricas cráneofaciales. Int. J. Odontostomat., 1:(2):165-8, 2007.

Dirección para correspondencia:

Prof. Dr. Iván Suazo Galdames

Departamento de Anatomía Normal

Universidad de Talca

Avenida Lircay s/n Oficina $N^{\circ} 104$

CHILE

Received : 28-09-2008

Email: isuazo@utalca.cl 